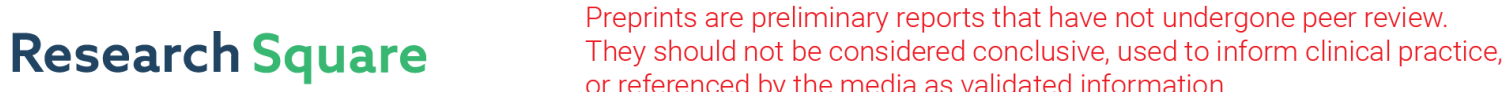 or referenced by the media as validated information. \\ Circularly-polarized THz wave emission from a micro-thin water flow
}

\author{
Hsin-hui Huang
}

Academia Sinica

\section{Saulius Juodkazis}

Swinburne University of Technology https://orcid.org/0000-0003-3897-2844

\section{Eugene Gamaly}

Australian National University

\section{Takeshi Nagashima}

Setsunan University

\section{Tetsu Yonezawa}

Hokkaido University https://orcid.org/0000-0001-7371-204X

Koji Hatanaka ( $\nabla$ kojihtnk@gate.sinica.edu.tw)

Academia Sinica

\section{Article}

Keywords: water-based THz emission, pulse irradiation, non-ionizing radiation

Posted Date: October 11th, 2021

DOI: https://doi.org/10.21203/rs.3.rs-77877/v2

License: (c) (i) This work is licensed under a Creative Commons Attribution 4.0 International License.

Read Full License 


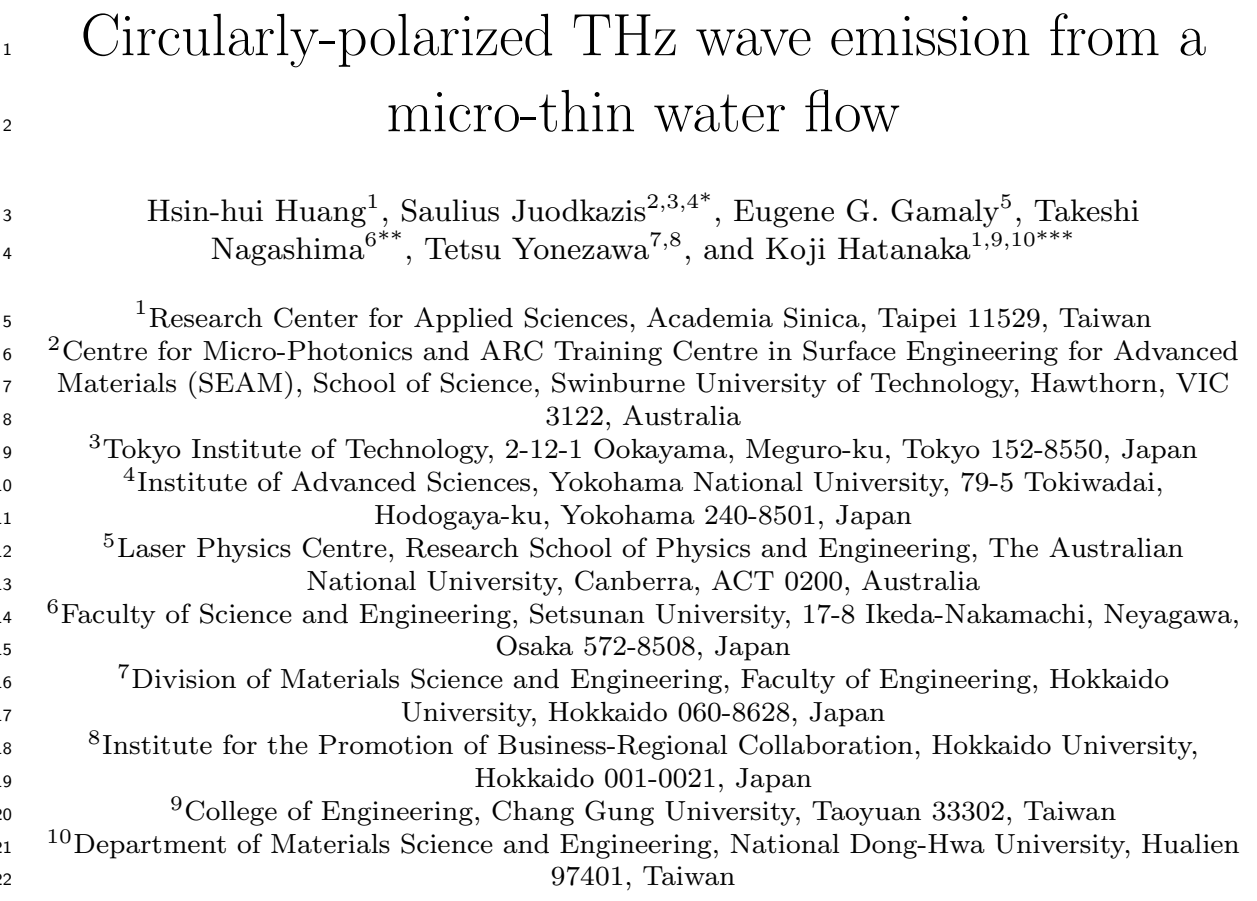

Intense $\mathrm{THz}$ wave sources are highly expected for further progresses in nonlinear $\mathrm{THz}$ science and practical implementation of non-ionizing radiation in sensing and communications. Solid-based sources have inherent limits of material breakdown, while intense laser irradiation of liquids is a promising emerging technique for $\mathrm{THz}$ wave and hard X-ray emission. Water-based THz emission shows intensity enhancements up to $10^{3}$ times when laser-pulse pairs with nanosecond delay are used. Here we show circularly-polarized $\mathbf{T H z}$ wave emission from thin water flow irradiated by two time-separated and linearly-polarized femtosecond laser pulses. $\mathrm{THz}$ time-domain spectroscopy reveals the circularly-polarized $\mathrm{THz}$ emission dominates $4.7 \mathrm{~ns}$ after the first pulse irradiation. $\mathrm{THz}$ wave detection delay in the spectroscopy and time-resolved micrography indicate that the $\mathrm{THz}$ wave emission originates from the rarefied volume in front of the flow. Radial relaxation of charges in the focal volume where ponderomotive charge depletion occurred on the optical axis is the origin for the circular polarization (due to spiraling currents). Tight focusing of fs-laser pulses localized $\mathrm{THz}$ wave emission to the sub-wavelength (tens-of-micrometers) region. 
Along with the fertile progresses in $\mathrm{THz}$ science and technology, intense and tunable $\mathrm{THz}$ wave radiation has been expected for advanced applications such as non-linear $\mathrm{THz}$ science [1]. Though there are various types of $\mathrm{THz}$ wave sources from electrically-driven devices to femtosecond laser methods based on optical rectification with nonlinear solid crystals and laser-plasma with targets of gases and clusters [2]. The solid targets have their own inherent limitations for the incident laser power because of the target irreversible damages, therefore scaling up intense $\mathrm{THz}$ wave radiation cannot be achieved. Since liquids such as water is a renewable target, it can be used under intense laser irradiation for THz emission [3-5] simultaneously with X-ray emission from hot plasma [5]. Water has another characteristic as a universal polar solvent, which will expand the tunability of $\mathrm{THz}$ wave sources [6].

Furthermore, not only the $\mathrm{THz}$ intensity but its polarisation is important. Circularly-polarised $\mathrm{THz}$ sources are required in the study of laser filamentation $[7,8]$, spin dynamics of solid state materials [9], chirality of proteins [10]. In material sciences, the state of angular momentum of light, i.e., its polarisation, effects the optical excitation [11], spectroscopy [12-14], sensing and communications [2], and provides data transfer multiplexing in information technologies [15]. However, there is lack of low loss polarisation optical waveplates for $\mathrm{THz}$ spectral range, what makes generation methods of circularly polarised $\mathrm{THz}$ sought after.

There are reports of circularly-polarized $\mathrm{THz}$ wave emission from plasma under a two-color laser excitation [16-20]. The ellipticity of the THz wave can be controlled by the filament length with suitable input laser energy [21], the relative phase between the fundamental wave and the second harmonic pulses [22-24], the pulse energy [25], and changing the time delays between three-pulse configuration [26]. Polarisation of $\mathrm{THz}$ wave emission from the filament of optically induced plasma was described by considering a dipole radiation inside $\sim 1 \mathrm{~mm}$ water jets [27,28] or in air [29] oriented along the propagation of femtosecond laser pulses and formed as a result of ponderomotive force. The ponderomotive force behind the ionisation front of a self-guided femtosecond laser pulse in air created a $L=1-30 \mathrm{~cm}$ long, $\sim 0.1 \mathrm{~mm}$ diameter filaments which induced $\mathrm{THz}$ wave emission with a spatial intensity and polarisation pattern defined by a moving dipole mechanism [29]. The polarisation of $\mathrm{THz}$ wave emission was found to be linear-radial (in respect to the propagation axis) as revealed by the squared Malus dependence $\propto \sin ^{4} \theta$. In this study, we investigate the case when the filament length, $L$, is sub-wavelength (sub-1 mm) at the tight focusing when $L$ corresponds to the geometrical focus, i.e., the Rayleigh length (see, the concept illustration in Fig. 1). Tight focusing of ultrashort laser pulses has additional benefit for the higher $\mathrm{THz}$ wave emission due to scaling for the ponderomotive force induced wake field dipoles, $P_{T H z}=\frac{1}{7}\left(\frac{E_{p}}{w_{0}}\right)^{2}\left(\frac{\lambda}{t_{p}}\right)^{4}$, where $w_{0}=0.61 \lambda / N A$ is the waist of the laser beam at the focus (a lens with numerical aperture $N A$ ), $t_{p}$ is pulse duration and $\lambda$ is its wavelength [30]. THz wave emission from sub-wavelength filaments is not yet explored research area which is strongly required for ultimate control of intensity, direction, phase, and 


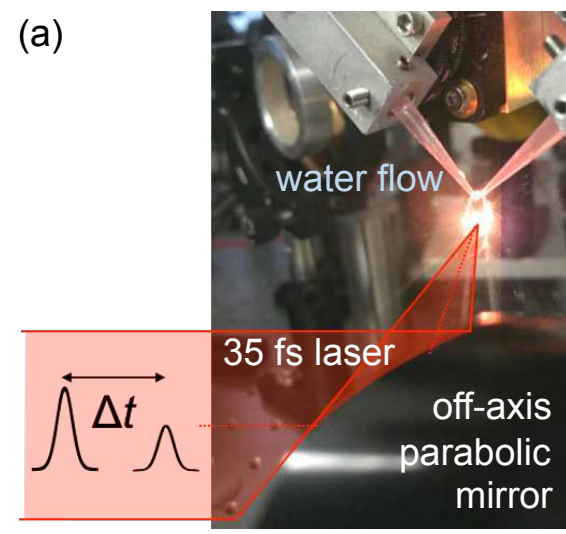

(c)

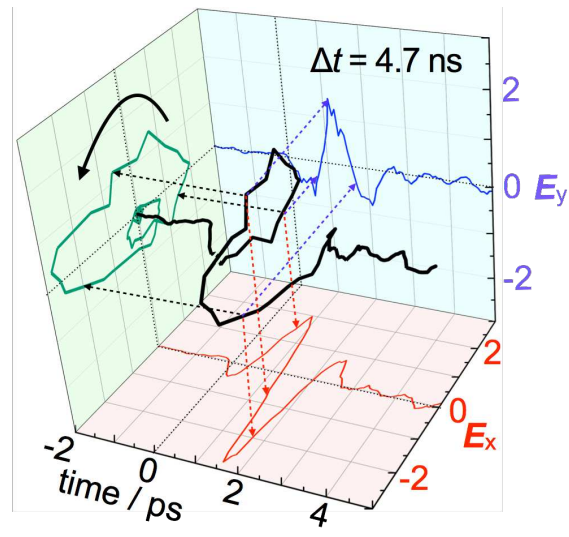

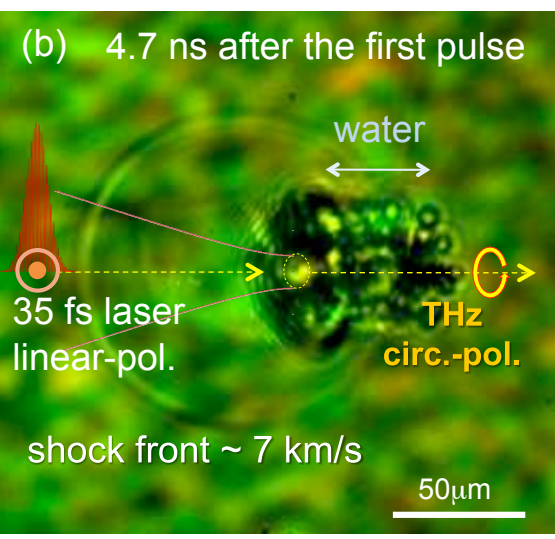

(d)

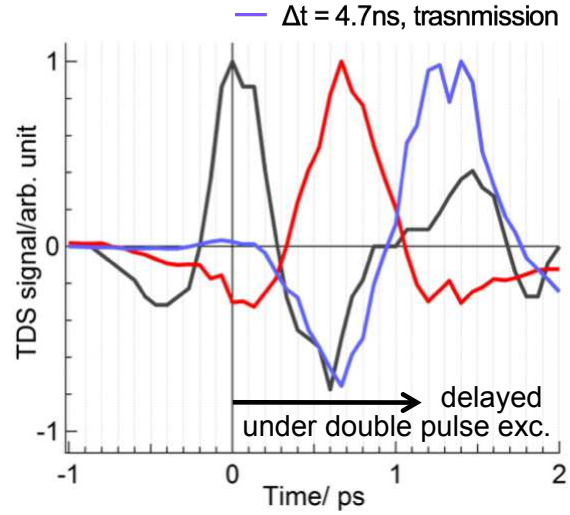

Figure 1: Concept: tailoring the light-matter interaction for the polarization manipulation of $\mathrm{THz}$ wave. (a) A thin water flow irradiated by a pair of femtosecond laser pulses (35fs, 800nm) for $\mathrm{THz}$ wave generation. Pulses are tightly focused to a $\sim 10 \mu \mathrm{m}$ spot on the surface of a $17 \mu \mathrm{m}$-thick water flow. (b) Time-resolved shadowgraphy with $\sim 1$ ps temporal resolution; a representative photo captured at $4.7 \mathrm{~ns}$ after the first pulse irradiation. Details are in Suppl. S1.2 and Fig. S2. (c) Signals in THz time-domain spectroscopy (TDS) when the delay time at $4.7 \mathrm{~ns}$, which indicates the circularly-polarized THz wave emission. Details are in Suppl. S1.1 and Fig. S1. (d) TDS time delay observed under the double pulse excitation; here TDS measurements were not polarization-discriminated.

polarisation of $\mathrm{THz}$ wave sources.

Creation of single-cycle $\mathrm{THz}$ wave sources and to control their polarisation could open a versatile toolbox of polarisation sensitive characterisation techniques currently developed at a rudimentary level. This study shows a promising direction towards generation of a single cycle circularly-polarised $\mathrm{THz}$ wave from focus of femtosecond laser pulse with sub-wavelength extension (for $\mathrm{THz}$ wave). Small sub-wavelength $\mathrm{THz}$ wave emitters provide flexibility in engineering $\mathrm{THz}$ 
wave sources with complex wavefronts, polarisation, and high power currently achieved by millimeters-long filaments [31]. Here, we report circularly-polarized $\mathrm{THz}$ wave emission from micro-thin water flow under double-pulse cross-linearlypolarized laser excitation. Axial extension of the $\mathrm{THz}$ wave-emitting filament of the second pulse (the main pulse) is enclosed inside the expanding water plume created by the first pulse (the pre-pulse) and has approximately the length of focal region of $60 \mu \mathrm{m}$ at the focusing conditions. The most efficient $\mathrm{THz}$ wave emission corresponded to the pulse separation of 4.5-5 ns. A current generated to restore charge depletion along the optical axis due to action of the ponderomotive force with simultaneously occurring recombination between ions and electrons is 1-2 ps long defining the spectrum of $\mathrm{THz}$ wave emission. Detailed polarisation analyses of $\mathrm{THz} E$-fields in the transmission and reflection directions are carried out by time-domain spectroscopy revealing peculiarities of sub-wavelength $\mathrm{THz}$ wave emission. The region of $\mathrm{THz}$ wave emission is also captured by time-resolved shadowgraphy/luminescence imaging experiments which confirm the origin of $\mathrm{THz}$ wave emission is in front, but not inside, of the water flow (Fig. 1).

\section{Results}

Circularly-polarised THz wave emission. Figure 1(c) shows polarisation resolved emission of $\mathrm{THz}$ wave at the optimum conditions with delay between two pulses of $4.7 \mathrm{~ns}$ using time-domain spectroscopy (TDS); more details are described in Suppl. S1.1 with Fig. S1 where the $E_{x}$ and $E_{y}$ are measured in the transmission and the reflection directions. $\mathrm{THz}$ wave emission under the single pulse excitation maintains the same $E_{x}$ polarization with the main pulse. However, under the double pulse excitation, $\mathrm{THz}$ wave emission changes its polarization from linear to circular as the delay time advances and its intensity reaches the highest at the delay time of $4.7 \mathrm{~ns}$ as reported previously without detailed polarisation analysis [32]. $\mathrm{THz}$ wave emission spectra obtained from TDS signals by discrete Fourier-transform, as shown in the inset in Fig. S1, show their peak shifts toward the lower frequency as the delay time increases. This indicates that the the interaction length of the main pulse $E_{2}$ with the ablated water plume after the pre-pulse $E_{1}$ irradiation becomes longer.

Direct imaging of light-water interaction. Figure 1(b) shows the timeresolved shadowgraphy image captured by back-side illumination of picosecond white light continuum at the maximum enhancement of $\mathrm{THz}$ wave emission. It clearly defines the geometry of the focal spot which is important in the estimation of light-matter conditions in the case on $\mathrm{THz}$ wave emission. The focus diameter on the flow was close to the geometrical focus with the radius $\sim 0.61 \lambda_{l} / N A \approx 4 \mu \mathrm{m} ; N A=0.125$ is the effective numerical aperture of the off-axis parabolic mirror. This is markedly different from $\mathrm{THz}$ wave emission in self-guided filament formation in air $(\sim 100 \mu \mathrm{m}$ diameter and centimeters-long $)$ governed by self-focusing of femtosecond laser pulses [29]. Detailed evolution 
of micro-explosion is presented in details in shadowgraphs (Fig. S2 in Suppl. S1.2) revealing the evolution of the shockwave induced by the pre-pulse irradiation. Shockwave expansion velocity in air was approaching $7.6 \mathrm{~km} / \mathrm{s}$, which corresponds to strong explosion conditions. The radius of of the shocked hemisphere in front of the water flow is $R \approx 60 \mu \mathrm{m}$ (at the delay time of $4.7 \mathrm{~ns}$ ) when the diameter of laser on the water is $w_{0} \approx 8 \mu \mathrm{m}$ and depth of ablation is $h \approx 2 \mu \mathrm{m}$. The additional ablated volume of water constitutes only $0.02 \%$ of volume in the shocked region, however, the molecular number density of water is $1.237 \times 10^{3}$ larger as compared with air. As a result, the molecular density of the water-air is at least by $26 \%$ larger as it is for the first pulse. This allows the plasma to reach higher density by the second pulse $E_{2}$. Indeed, as shown in the luminescence images (Fig. S2 in Suppl. S1.2), interaction of the main pulse with water flow previously modified by the pre-pulse irradiation becomes more dominant. The very central part of light-matter interaction on the flow surface is the source of X-ray emission since the electron temperature reaching $\sim 1 \mathrm{keV}$ is achieved under the experimental conditions [5,32] (see Suppl. S1.3; however, this study was solely focused on $\mathrm{THz}$ wave emission).

Figure 1(d) shows the time delay in TDS signals observed at the different excitation conditions. The TDS signals under the double pulse excitation delay for $\sim 700 \mathrm{fs}$ in the reflection and for $\sim 1.4 \mathrm{ps}$ in the transmission. By considering that the refractive index of water at $1 \mathrm{THz}$ is $\sim 2.12$ [33], it is consistent with direct imaging and indicates that the conversion from the near-IR laser to $\mathrm{THz}$ wave takes place in front of the original water flow surface. This observation indicates that the ablation of water by the pre-pulse $E_{1}$ and the shockwave dynamics is important for the $\mathrm{THz}$ wave emission under the double pulse excitation. The location of $\mathrm{THz}$ wave emission is presented earlier to originate from the inside of water flow [34] which is in this case highly unlikely due to extraordinary-high plasma density (water molecular density $3.35 \times 10^{22} \mathrm{~cm}^{-3}$ ) for the ponderomotive mechanism under employed intensity range.

Radial charge transport at the tight focus. Figure 2 shows the key features on the light-matter interaction relevant for the circularly-polarised $\mathrm{THz}$ wave emission. The second/main pulse $E_{2}$ ionises the outwardly fast expanding gas and the water vapor generated by the pre-pulse $E_{1}$. The most intense $\mathrm{THz}$ wave emission is observed at $\Delta t=4.7 \mathrm{~ns}$. This corresponds to the fast $(\sim 1 \mathrm{ps})$ currents along the optical path of the main pulse $E_{2}$, which are due to electrons and ions radially depleted along the propagation due to the ponderomotive force which for electron is $F_{p}=-\frac{e^{2}}{4 m_{e} \omega^{2}} \nabla I$, where $I$ is the laser intensity. The radial profile of the electronic density depletion on the axis of a Gaussian pulse $I(r)=I_{0} e^{-4 \ln 2\left(r / r_{0}\right)^{2}}$ is given by $N_{e}(r) / N_{0}=1+\left(\frac{d^{2}}{d r^{2}}+\frac{1}{r} \frac{d}{d r}\right) \sqrt{1+I^{2}(r)}$ where $N_{e, 0}$ is the electron and background densities, respectively, $r_{0}$ is the FWHM of the focal spot. The radial electron displacement has a steeper gradient as compared with the axial charge separation due to ponderomotive force due to Gaussian intensity envelope. This is the key difference from the self-guided long filaments which never produced circularly-polarised $\mathrm{THz}$ wave emission 


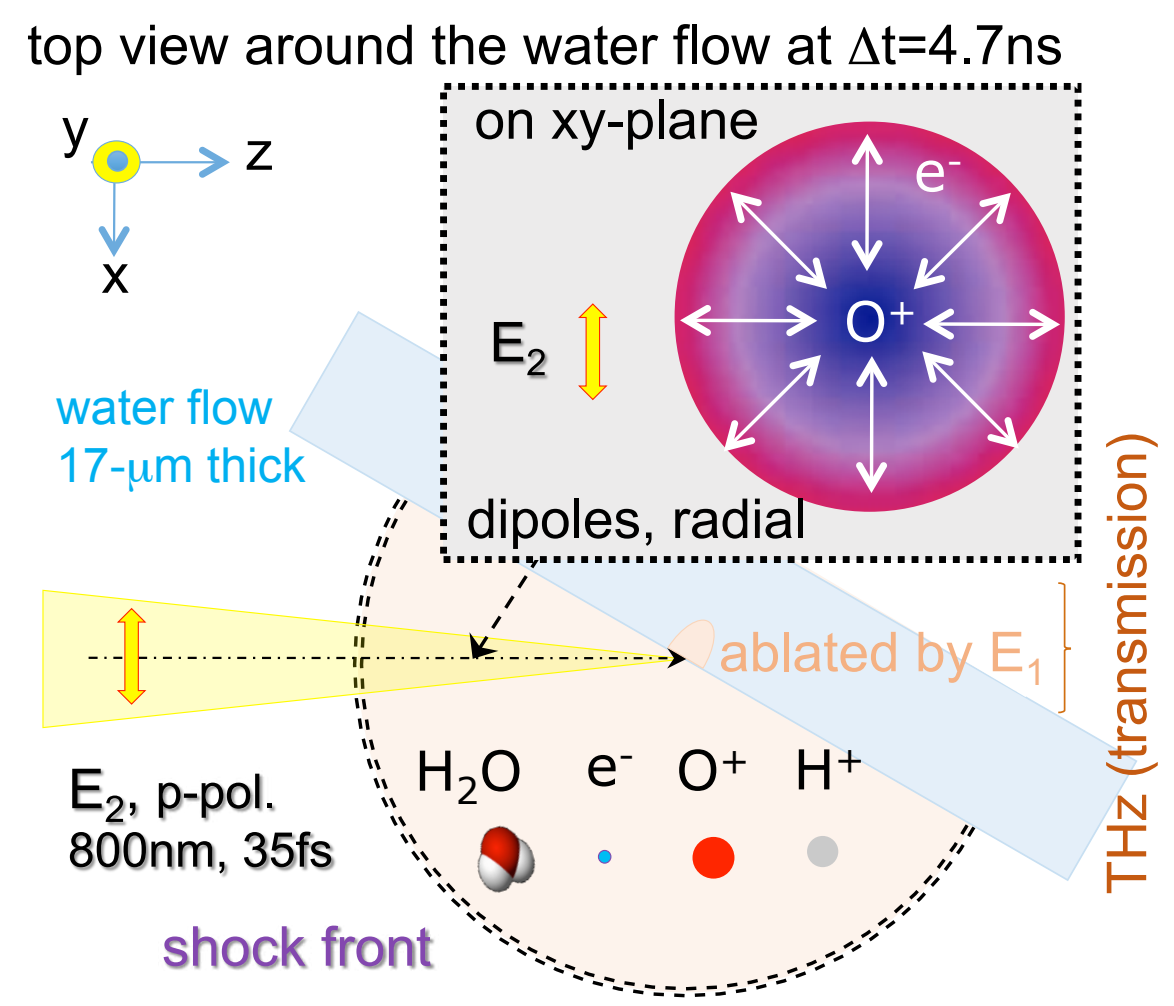

Figure 2: Geometry of the experiment for the $N A=0.125$ focusing depicted after the pre-pulse $E_{1}$ (y-pol.) irradiation to the water flow which creates the expanding plasma region out of the ablated water flow (strong explosion with shockwave of radius $r_{s h}$; see Suppl. S1.2). THz wave emission is induced by the current of ionised plasma rarefied along the center axis of the propagating $E_{2}$ laser pulse due to the radial ponderomotive force. The transient radial current $\partial J / \partial t$ is the source of $\mathrm{THz}$ wave emission. The Rayleigh length under the focusing conditions is $z_{R} \approx 60 \mu \mathrm{m}\left(2 z_{R}\right.$ is the depth-offocus at 0.5 -intensity level, i.e. FWHM), which would correspond to the $\lambda_{T H z} / 4$ for the emission at $1.25 \mathrm{THz}(240 \mu \mathrm{m})$.

since the radiating dipole (via Cherenkov-like mechanism) is axially oriented. The radial pattern of arrows in the xy-plane cross-section shows charge separation by the ponderomotive force (the radial pattern will occur for electrons, protons $\mathrm{H}^{+}$and $\mathrm{O}^{+}$which will have different radial distribution due to their difference in mass; see Suppl. S1.3). The radial ion-electron charge diffusion and recombination onto the optical axis takes place in the wake of the optical pulse. This ultrafast current transient is the source of $\mathrm{THz}$ wave emission which is phase-tailored into circularly-polarised radiation as the second pulse ( $35 \mathrm{fs}$ or $10 \mu \mathrm{m}$ ) ionised the $\sim 60 \mu \mathrm{m}$ long focal region. The current was initiated at the locations further away from the focus and propagated towards the water 
flow. It was shown that the circularly spiraling current will generate circularlypolarised THz radiation when spiral electrodes were used [35]. Previous studies with linearly-polarised light pulses have shown that in the case of irradiation of Ar cluster in vacuum by two time-separated pulses, the polarisation of the $\mathrm{THz}$ wave emission was defined along the line through the center of the first pulse and the position of the focus of the second pulse (which was shifted out of center by $\sim 40 \mu \mathrm{m}$ ) [36]. The intensity of the first pulse was inducing the charge depletion on the axis with positive ions closer to the center (on the optical axis). Electrons generated out-off-the-center by the second pulse caused a linear current (towards the center) and consequently linearly-polarised $\mathrm{THz}$ wave emission. The conditions of tight focusing explored in our study creates spatially confined plasma at the focal region along the optical axis. Since electrons, protons, and oxygen ions from the water flow (and oxygen and nitrogen from air) are separated radially to the different position by the pre-pulse, the second pulse initiates a spiraling current along the depth-of-focus (more details are discussed in Suppl. S1.4). The irradiation position of the first pulse to that of the main pulse is optimised for the strongest $\mathrm{THz}$ wave emission by the same methodology as we described earlier for the maximum of X-ray emission [32]; i.e., slight walk-off on the xy-plane is introduced for the maximum of the emission. This optimisation is obtained for approximately $10 \mu \mathrm{m}$ shift along the $\mathrm{x}$-axis on the flow surface. Quantification of the offset from the optical axis for the intensity and polarisation control of $\mathrm{THz}$ wave emission needs further studies. It is established in this study that the circularly-polarised single cycle $\mathrm{THz}$ pulse can be generated using the tight optical-focusing from ionised focal volume in the pre-plasma with the axial extent of $\lambda / 4$ of $\mathrm{THz}$ wave emission.

\section{Conclusions}

A simple two-laser pulse irradiation to a thin water flow is shown to generate circularly-polarized $\mathrm{THz}$ wave emission from a plasma filament which has length of approximately quarter wavelength of the $\mathrm{THz}$ emission (a sub-wavelength $\mathrm{THz}$ emitter). The mechanism responsible for the increased efficiency of $\mathrm{THz}$ wave emission as compared with the single pulse irradiation of solid/liquid/gas targets is related to water ablation inside strong shock region in air in front of the water flow. The ablation condition for $\mathrm{THz}$ wave emission is optimal at the delay time $4.7 \mathrm{~ns}$ after the pre-pulse irradiation. The radial current transients restoring axially depleted charges due to action of ponderomotive force determines the spectral extent (a shorter transient broader spectrum as related via Fourier transform) and the circular polarisation of such $\mathrm{THz}$ wave emission. Circular polarisation is a result of radially spiraling currents which originate along the $\lambda_{T H z} / 4$ long Rayleigh region of optical focus (a linearly-polarised THz wave are observed from the axially oriented dipole in self-guided long filaments [29]). $\mathrm{THz}$ wave emission in the reflection and transmission directions in respect to the water flow has similar intensities but opposite polarisation. The transmission losses of sub-mm wavelength $\mathrm{THz}$ through $17-\mu \mathrm{m}$-thick water flow are slightly 
reducing the transmitted power. As previously reported [32], the highest intensity of $\mathrm{THz}$ wave emission from the water flow under the double pulse excitation is 10-times higher in $|\boldsymbol{E}|^{2}$ (as photon numbers) than that from a $\langle 110\rangle$-oriented ZnTe crystal. Further enhancements are expected in other liquids such as gold nano-colloidal aqueous suspensions.

Circularly-polarised $\mathrm{THz}$ wave emission is adding new possibilities in a polarisation control toolbox for $\mathrm{THz}$ technologies which can open new applications for opto-mechanics where large torsion can be generated upon absorption, reflection, or scattering and for rotational/vibrational molecular spectroscopy where polarisation wave-plates are not readily available or lossy. $\mathrm{THz}$ wave can be used to better understand highly dynamic laser triggered explosions which find increasing number of application in laser machining/fabrication and creation of high temperature/pressure phases of new materials. Among different methods of electromagnetic field generation, sub-wavelength emitters are promising due to unmatched flexibility in wave front and polarisation engineering.

\section{Methods}

All the experiments shown in Fig.3 were carried out in air under atmospheric pressure $(1 \mathrm{~atm})$ at room temperature $(296 \mathrm{~K})$.

Polarization-sensitive THz time-domain spectrosocpy. A pulsed femtosecond laser $\left(t_{p}=35 \mathrm{fs}\right.$, transform-limited, $\lambda=800 \mathrm{~nm}, 1 \mathrm{kHz}$, Mantis, Legend Elite HE USP, Coherent, Inc.,) is used and the output pulses are split into the pre-pulse $\left(E_{1}\right.$, linearly-polarized parallel to y-axis, y-pol., 0.1 or 0.2 $\mathrm{mJ} /$ pulse), the main pulse $\left(E_{2}\right.$, linearly-polarized parallel to x-axis, x-pol., 0.4 $\mathrm{mJ} /$ pulse), and the probe for $\mathrm{THz}$ time-domain spectroscopy (TDS) with a series of half-wave plates and polarization beam splitters (65-906, 47-048, Edmund Optics) as shown in Fig. 2 (a) [5,6,32]. THz wave emission is induced by the irradiation of the co-linearly combined pre-pulse and main pulse onto a thin water flow $(\sim 17 \mu \mathrm{m}$ thick) by an off-axis parabolic mirror (1-inch diameter, effective focal length $f=50.8 \mathrm{~mm}, 47-097$, Edmund Optics). The laser incident angle along z-axis is at $60^{\circ}$ to the water surface normal. Under this condition,the polarisations of the main and the pre-pulses are p-pol. and s-pol., respectively. The optical delay between the two excitation pulses, $\Delta t$, is controlled with automatic stages (SGSP46-800 and SGSP26-150, Sigma Koki). A water flow is prepared wth two colliding water jets and the system is set on an automatic stage (KS701-20LMS, Suruga Seiki) along the z-axis to adjust the flow surface for optimal X-ray emission measured by a Geiger counter (SS315, Southern Scientific). The detection of the $\mathrm{THz}$ wave emission is carried out by the electro-optic sampling method in the transmission direction through the water flow and in the reflection direction with $\langle 110\rangle$-oriented ZnTe crystals (1-mm thick, Nippon Mining \& Metals Co., Ltd.). Lock-in measurements are carried out with an optical chopper (3502, New Focus) and a lock-in amplifier (SR830, Stanford Research System), therefore the effective repetition rate of the laser excitation is $0.5 \mathrm{kHz}$. Following the usual method reported previously [37-39], 


\section{(a) THz time-domain spectrosocpy}

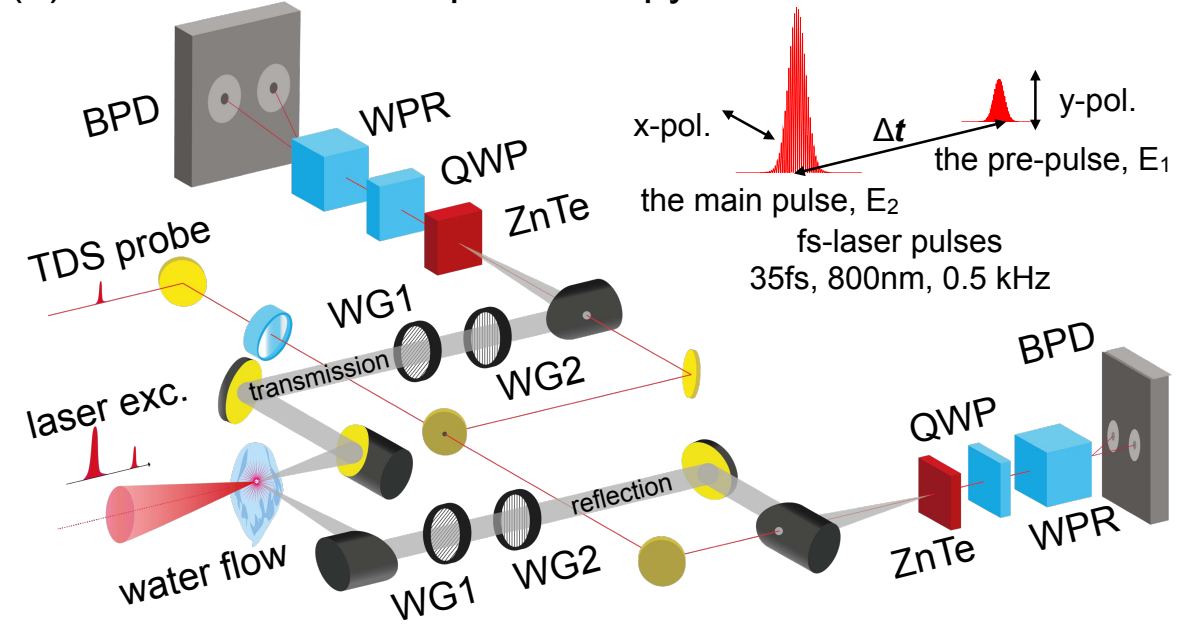

(b) imagings

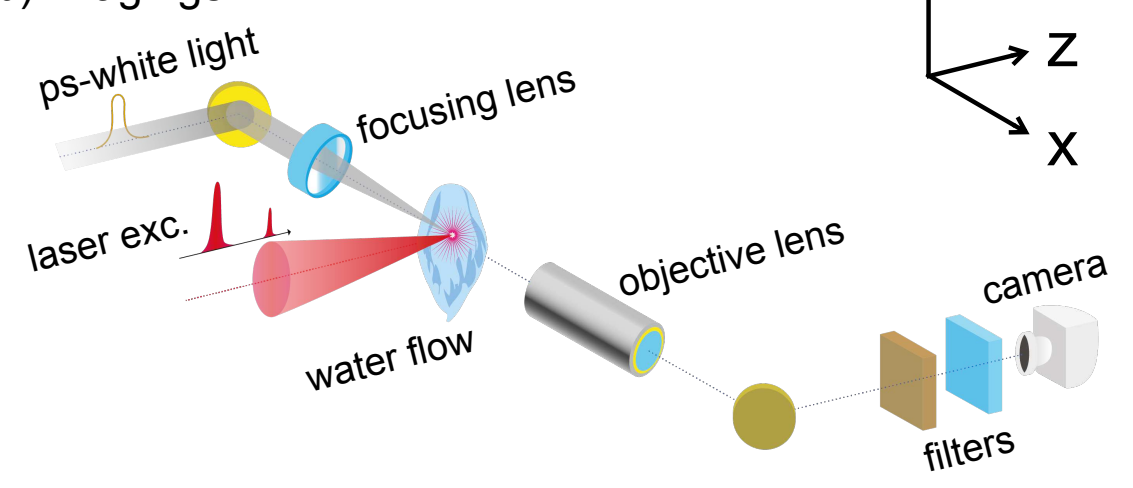

Figure 3: Schematic diagrams of the experimental setups with the water flow (17 $\mu \mathrm{m}$ thick) under the tight focusing of double pulses with the pre-pulse (x-pol., $E_{1}$ ) and the main pulse (y-pol., $E_{2}$ ) (a) for $\mathrm{THz}$ time-domain spectroscopy (TDS) with a ZnTe crystal (for electro-optic sampling) and (b) for time-resolved shadowgraphy and luminescence imaging. $\Delta t$ indicates the delay time between the two pulses. Polarization statuses of $\mathrm{THz}$ wave emission were measured with a pair of wire-grids. Imaging experiments for the laser focus were also carried out with an objective lens and a color camera with the exposure time at $2 \mathrm{~ms}$ for the single shot. The details are in the text. QWP:quarter wave-plate, WPR:Wollaston prism, BPD:balanced photo-diode

two wire grids (WGs, MWG40FA-III, Origin) are additionally used for the measurements of polarization status in THz wave emission. One of the WGs, WG2, is used at the fixed angle $0^{\circ}$ (parallel to x-axis), while the other WG, WG1, is used at two different angles, $+45^{\circ}$ and $-45^{\circ}$ for two independent TDS signals as $E_{+45}$ and $E_{-45}$, respectively. The x- and y-components of $\mathrm{THz}$ electric field, $E_{x}$ 
and $E_{y}$, are then calculated from $E_{x}=E_{+45}+E_{-45}$ and $E_{y}=E_{+45}-E_{-45}$, respectively.

Time-resolved imagings. Imaging experiments for the laser focus from the side (along x-axis) are also carried out in two different methods with an objective lens (M Plan Apo 10×, MITUTOYO) and a color CMOS camera (Blackfly S USB3, FLIR Systems, Inc.) with filters for IR-cut and for intensity control in the visible region. One is with the pre-pulse and white light continuum $(\sim 1 \mathrm{ps}$, $580 \pm 30 \mathrm{~nm}$, as a strobe light) converted from the main pulse with a water cell. With this method, transient refractive index changes and/or scattering due to pre-plasma formation and/or laser ablation induced by the pre-pulse irradiation can be visualized. Another imaging is with the pre-pulse and the main pulse, which visualizes the interaction of the main pulse with the water flow with structures prepared by the pre-pulse irradiation. The exposure time for the camera setting was fixed at $2 \mathrm{~ms}$ for single shot imaging. In this mode of image acquisition, all the emission in broad-band spectra by the two-pulse irradiation of the water flow is time-integrated.

\section{References}

[1] K. Tanaka, H. Hirori, M. Nagai, IEEE Transactions on Terahertz Science and Technology 2011, 1, 1301.

[2] J. A. Fülöp, S. Tzortzakis, T. Kampfrath, Advanced Optical Materials 2020, 8, 31900681.

[3] I. Dey, K. Jana, V. Y. Fedorov, A. D. Koulouklidis, A. Mondal, M. Shaikh, D. Sarkar, A. D. Lad, S. Tzortzakis, A. Couairon, G. R. Kumar, Nat. Commun. 2017, $\underline{8}, 11184$.

[4] J. Qi, E. Yiwen, K. Williams, J. Dai, X.-C.Zhang, Appl. Phys. Lett. 2017, $\underline{111}, 7071103$.

[5] H.-H. Huang, T. Nagashima, W.-H. Hsu, S. Juodkazis, K. Hatanaka, Nanomaterials 2018, $\underline{8}, 7523$.

[6] H.-H. Huang, Y.-t. R. Chau, T. Yonezawa, M. T. Nguyen, S. Zhu, D. Deng, T. Nagashima, K. Hatanaka, Chemistry Letters 2020, 49, 6597.

[7] L. Bergé, S. Skupin, C. Köhler, I. Babushkin, J. Herrmann, Phys. Rev. Lett. 2013, 110073901.

[8] L. Bergé, J. Rolle, C. Köhler, Phys. Rev. A 2013, 88023816.

[9] A. S. Prokhorov, V. B. Anzin, D. A. Vitukhnovskiı̌, E. S. Zhukova, I. E. Spektor, B. P. Gorshunov, S. Vongtragool, M. B. S. Hesselberth, J. Aarts, G. J. Nieuwenhuys, M. Dumm, D. Faltermeier, S. Kaiser, S. Yasin, M. Dressel, N. Drichko, Journal of Experimental and Theoretical Physics 2006, 103, 6887 . 
[10] X. Yang, X. Zhao, K. Yang, Y. Liu, Y. Liu, W. Fu, Y. Luo, Trends Biotechnol. 2016, 34810.

[11] T. L. Cocker, V. Jelic, M. Gupta, S. J. Molesky, J. A. J. Burgess, G. D. L. Reyes, L. V. Titova, Y. Y. Tsui, M. R. Freeman, F. A. Hegmann, Nature Photonics 2013, $\underline{7}, 8620$.

[12] J. Zhu, Z. Ma, W. Sun, F. Ding, Q. He, L. Zhou, Y. Ma, Applied Physics Letters 2014, 105, 2021102.

[13] B.-X. Wang, L.-L. Wang, G.-Z. Wang, W.-Q. Huang, X.-F. Li, X. Zhai, Applied Physics A 2014, 115, 41187.

[14] R. Singh, E. Plum, C. Menzel, C. Rockstuhl, A. K. Azad, R. A. Cheville, F. Lederer, W. Zhang, N. I. Zheludev, Phys. Rev. B 2009, 80153104.

[15] S. Baierl, M. Hohenleutner, T. Kampfrath, A. K. Zvezdin, A. V. Kimel, R. Huber, R. V. Mikhaylovskiy, Nature Photonics 2016, 10, 11715.

[16] D. J. Cook, R. M. Hochstrasser, Opt. Lett. 2000, 25, 161210.

[17] M. Kress, T. Löffler, S. Eden, M. Thomson, H. G. Roskos, Opt. Lett. 2004, 29, 101120 .

[18] H. Zhong, N. Karpowicz, X.-C. Zhang, Applied Physics Letters 2006, 88, 26261103.

[19] I. Babushkin, W. Kuehn, C. Köhler, S. Skupin, L. Bergé, K. Reimann, M. Woerner, J. Herrmann, T. Elsaesser, Phys. Rev. Lett. 2010, 105053903.

[20] Z. Zhang, N. Panov, V. Andreeva, Z. Zhang, A. Slepkov, D. Shipilo, M. D. Thomson, T.-J. Wang, I. Babushkin, A. Demircan, U. Morgner, Y. Chen, O. Kosareva, A. Savel'ev, Applied Physics Letters 2018, 113, 24241103.

[21] Z. Zhang, Y. Chen, S. Cui, F. He, M. Chen, Z. Zhang, J. Yu, L. Chen, Z. Sheng, J. Zhang, Nature Photonics 2018, 12, 9554.

[22] H. Wen, A. M. Lindenberg, Phys. Rev. Lett. 2009, 103023902.

[23] J. Dai, N. Karpowicz, X.-C. Zhang, Phys. Rev. Lett. 2009, 103023001.

[24] Y. S. You, T. I. Oh, K.-Y. Kim, Opt. Lett. 2013, 38, 71034.

[25] Y. Chen, C. Marceau, S. Génier, F. Théberge, M. Châteauneuf, J. Dubois, S. L. Chin, Optics Communications 2009, 282, 214283 .

[26] H. Wang, N. Li, Y. Bai, P. Liu, Z. Wang, C. Liu, Opt. Express 2017, 25, 2530987.

[27] X.-C. Zhang, F. Buccheri, Lith. J. Phys. 2018, 58, 11. 
[28] E. Yiwen, Q. Jin, A. Tcypkin, X.-C. Zhang, Appl. Phys. Lett. 2018, 113 181103.

[29] C. D'Amico, A. Houard, M. Franco, B. Prade, , A. Mysyrowicz, A. Couairon, V. T. Tikhonchuk, Phys. Phys. Lett. 2007, 98235002.

[30] H. Hamster, A. Sullivan, S. Gordon, R. W. Falcone, Phys. Rev. E 2007, 98235002 .

[31] A. Koulouklidis, C. Gollner, V. Shumakova, V. Fedorov, A. Pugžlys, A. Baltuška, S. Tzortzakis, Nature Commun. 2020, 11, 11.

[32] H.-H. Huang, T. Nagashima, T. Yonezawa, Y. Matsuo, S. H. Ng, S. Juodkazis, K. Hatanaka, Applied Sciences 2020, 10, 62031.

[33] J. Zhou, X. Rao, X. Liu, T. Li, L. Zhou, Y. Zheng, Z. Zhu, AIP Advances 2019, $9,3035346$.

[34] Q. Jin, J. Dai, E. Yiwen, X.-C. Zhang, Appl. Phys. Lett. 2018, 113261101.

[35] X. Lu, X.-C. Zhang, Phys. Rev. Lett. 2012, 108123903.

[36] K. Mori, M. Hashida, T. Nagashima, D. Li, K. Teramoto, Y. Nakamiya, S. Inoue, S. Sakabe, Applied Physics Letters 2017, 111, 24241107.

[37] P. Yeh, Optics Communications 1978, 26, 3289.

[38] F. Miyamaru, T. Kondo, T. Nagashima, M. Hangyo, Applied Physics Letters 2003, 82, 162568.

[39] N. Kanda, K. Konishi, M. Kuwata-Gonokami, Opt. Express 2007, 15, 18 11117.

\section{Acknowledgments}

S.J. is grateful for partial support by the ARC Discovery DP190103284, Linkage LP190100505 and JST CREST JPMJCR19I3 grants. T.N. is grateful for the support by JSPS KAKENHI Grant Number 20K05371. T.Y. is grateful for the support by Grant-in-Aid for Scientific Research for Fostering Joint International Research (B) (18KK0159). K. H. is grateful for the supports by the Ministry of Science and Technology (MOST) of Taiwan (107-2112-M-001-014-MY3), the Cooperative Research Program of "Network Joint Research Center for Materials and Devices", Nanotechnology Platform (Hokkaido University), and the Collaborative Research Projects of Laboratory for Materials and Structures, Institute of Innovative Research (Tokyo Institute of Technology). K. H. also acknowledges the Japan Science and Technology Agency (JST) PRESTO (Precursory Research for Embryonic Science and Technology) Program (SAKIGAKE, Innovative use of light and materials/life) for its supports on the original project on X-ray/ $\mathrm{THz}$ wave simultaneous emission, "Ultrawide band light conversion by controlling structures of microdroplets and ultrashort laser pulse (20092013)" and for the laser facilities for the current project. H. -H. H. and K. H. acknowledge Mr. Yung-Jie Huang for his drawings of the experimental setups. 


\section{Supplementary Files}

This is a list of supplementary files associated with this preprint. Click to download.

- THzPolNatCommsupplement.pdf 